\title{
CYTOCHROME OXIDASE ACTIVITY IN EXTRAOCULAR MUSCLES
}

\author{
Yoshinori DAKE AND Tsugio AMEMIYA \\ Department of Ophthalmology, Nagasaki University School of Medicine, Nagasaki 852
}

Received for publication October 3, 1991 and in revised form December 26, 1991

\begin{abstract}
Cytochrome oxidase activity in extraocular muscles was examined by the diaminobenzidine method and analyzed with a computed image analyzer. Two types of mitochondria, described by Noda and Kuwahara, were found in muscles incubated for 30,60 and $120 \mathrm{~min}$. The reactivity and proportion of the two types of mitochondria showed time-dependent changes between 30 and 60 min of incubation. However, no changes were found between 60 and $120 \mathrm{~min}$ of incubation. It was suspected that potential cytochrome oxidase activity was present during 60 to $120 \mathrm{~min}$ of incubation. Quantitative analysis showed that type 1 mitochondria had more cytochrome oxidase activity than type 2 mitochondria. These findings suggest that the activity and proportion of the two types of mitochondria might be useful as an index of functional activity in extraocular muscles.
\end{abstract}

Cytochrome oxidase is the terminal enzyme of the electron transfer chain and is located on the inner membrane and cristae of mitochondria. Cytochrome oxidase activity is identified as non-droplet precipitates (reactive products) resulting from oxidation of diaminobenzidine (DAB) on the inner membrane and intracristal space of mitochondria. The area occupied with reactive products on the inner membrane and in the intracristal space appears to be correlated with the intensity of cytochrome oxidase activity. Kageyama et al. $(2,3)$ demonstrated that cytochrome oxidase activity shows variation in the lateral geniculate nucleus and striate cortex and that this variation is associated with intracellular energy demand and function. Noda and Kuwahara (4) classified mitochondria into two types according to the location of the reactive product and compared the mitochondria patterns of preosteoclasts and osteoclasts. In order to study the energy supply of mitochondria in extraocular muscles, we planned the present experiment to identify by electron microscopy and computed image analysis the variation of cytochrome oxidase activity in the extraocular muscles.

Mailing address: Yoshinori Dake, M. D. Department of Ophthalmology, Nagasaki University School of Medicine, 7-1 Sakamoto-machi, Nagasaki 852 Japan.

\section{MATERIALS AND METHODS}

Tissue Preparation for Electron Microscopy

The eyeballs of mature female Wistar albino rats weighing $300 \mathrm{~g}$ were enucleated under pentobarbital anesthesia. The caput of extraocular muscles was removed from the eyeball and fixed with $2 \%$ glutaraldehyde in $0.1 \mathrm{M}$ phosphate buffer at $4^{\circ} \mathrm{C}$ for $10 \mathrm{~min}$. After being washed with cold $0.1 \mathrm{M}$ phosphate buffer for $90 \mathrm{~min}$, specimens were cut into $100 \mu \mathrm{m}$ thick slices with a vibratome. With the diaminobenzidine (DAB) method of Seligman et al. (5) slices were preincubated with $0.01 \%$ catalase for $10 \mathrm{~min}$ and incubated in a medium containing $1.0 \mathrm{mg} /$ $\mathrm{ml}$ of 3,3 'diaminobenzidine-tetra-hydrochloride salt, $1 \mathrm{mg} / \mathrm{ml}$ of cytochrome c, $0.1 \mathrm{mg} / \mathrm{dl}$ of catalase, $7 \%$ sucrose and $0.1 \mathrm{M}$ phosphate buffer at $37^{\circ} \mathrm{C}$ for 0,30 , 60 and $120 \mathrm{~min}$. After being washed again in cold $0.1 \mathrm{M}$ phosphate buffer for $30 \mathrm{~min}$, they were postfixed with $1 \%$ osmium tetroxide in $0.1 \mathrm{M}$ phosphate buffer. All blocks were dehydrated in a graded ethanol series and propylene oxide, and embedded in Luveak 812. Ultrathin sections were cut and examined in a Hitachi $\mathrm{H}-300$ without staining.

Measurement of Reactive Products

Reactive products in mitochondrial intermembrane-intracristal spaces tend to grow into these spaces. The area of mitochondrial intermembrane-intracristal space in electron micrographs at a magnification of 70,000 was measured with a computed image 
analyzer, Zeiss IBAS, by a point count method (1). The area occupied by deposits of reactive products under an adequate threshold was measured by the same method. Tangential sections of mitochondria were eliminated from the measurement. The percentage of the area occupied by reactive products in each mitochondrion was calculated and analyzed statistically.

Noda and Kuwahara classified mitochondria into two types: Type 1, mitochondria with reactive products in inner membranes and outer compartments; Type 2 , mitochondria with reactive products only in the inner membrane of the cristae, or without reactive products at any site. On the basis of this classification, we analyzed the results.

\section{RESULTS}

At 0 min of incubation, no reaction products were seen (Fig. 1). At $30 \mathrm{~min}$ of incubation, a large number of mithochondria had reactive products only on the inner membrane of the cristae or no droplets at any site (Fig. 2). These products were not uniformly deposited on the inner membrane and their intensity was weak. Some mitochondria showed reactive products on the inner membrane of the cristae, intracristal space and outer compartment like type 1 mitochondria. At $60 \mathrm{~min}$, type 1 mitochondria were increased in number and reactive products filled the in-
TABle 1. Percentage of type 1 mitochondria in $25 \times 25 \mu \mathrm{m}$

\begin{tabular}{cc}
\hline Incubation time & \\
\hline $30 \mathrm{~min}$ & $36.2 \pm 21.9 \% * * * \quad(\mathrm{n}=25)$ \\
$60 \mathrm{~min}$ & $55.8 \pm 19.9 \% * \mathrm{~N} \quad(\mathrm{n}=25)$ \\
$120 \mathrm{~min}$ & $63.3 \pm 18.7 \% * * \mathrm{~N} \quad(\mathrm{n}=25)$ \\
\hline
\end{tabular}

$*(\mathrm{p}<0.01)$

$* *(\mathrm{p}<0.01)$

$\mathrm{N}$ no statistically significant difference

TABLE 2. Activity of cytochrome oxidase (\%)

\begin{tabular}{ll}
\hline Type 1 mitochondria & $82.4 \pm 12.7 \% \%^{*}(\mathrm{n}=30)$ \\
Type 2 mitochondria & $15.6 \pm 8.8 \%^{*}(\mathrm{n}=30)$ \\
\hline${ }^{*}(\mathrm{p}<0.01)$ &
\end{tabular}

tracristal space. Type 2 mitochondria were decreased in number. However, reactive products on the inner membrane were increased, and mitochondria with no deposits were fewer (Fig. 3). In specimens incubated for $60 \mathrm{~min}$, reactive products in type 2 mitochondria were almost the same as in those incubated for $120 \mathrm{~min}$ (Fig. 4). The percentage of type 1 mitochondria in a certain area $(25 \times 25 \mu \mathrm{m})$ was calculated in each incubation group and statistically examined with a

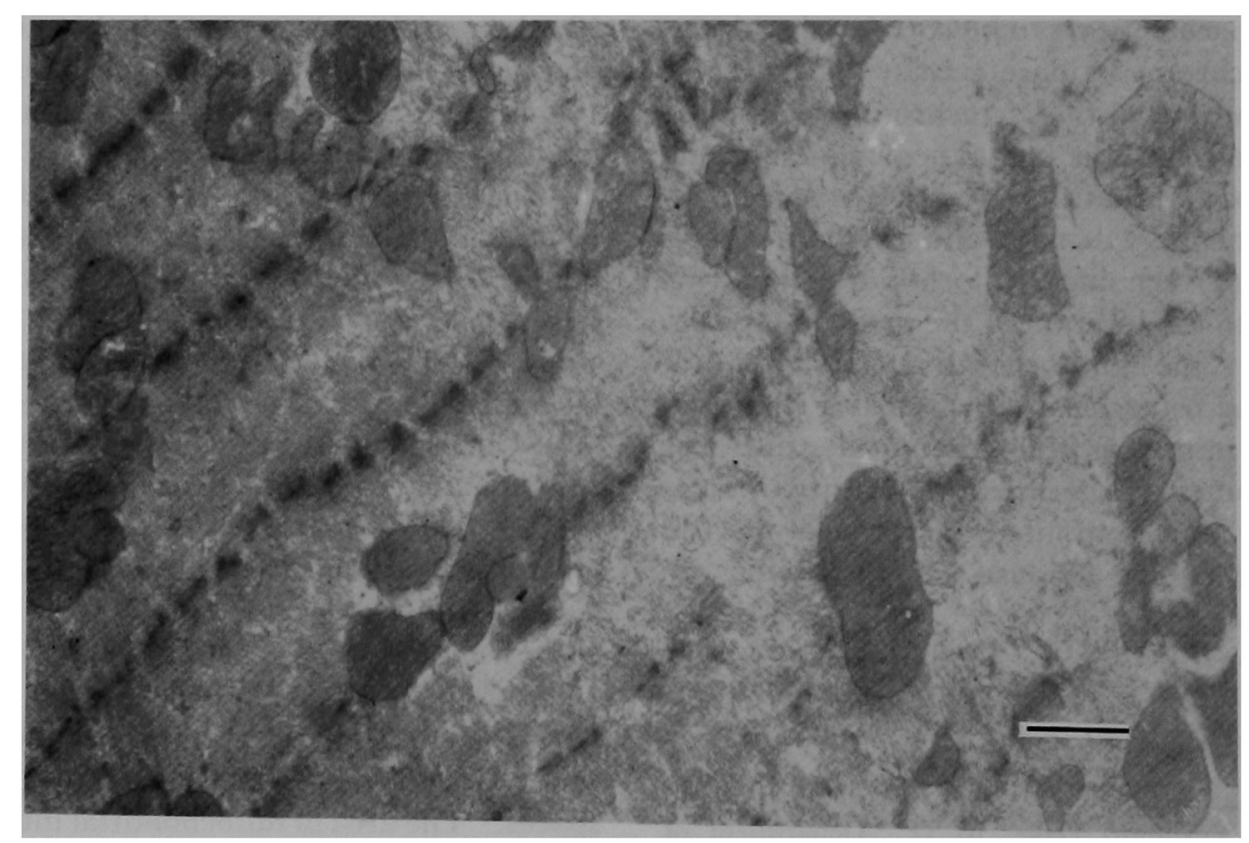

Fig. 1. Electron micrograph of extraocular muscle at $0 \mathrm{~min}$ of incubation. No reactive products were seen on the inner membrane and intracristal space of mitochondria. $\times 11,000 \quad \mathrm{Bar}=1 \mu \mathrm{m}$. 
paired $t$-test. The results are shown in Table 1 . There was no difference in mitochondria types between the $60 \mathrm{~min}$ and $120 \mathrm{~min}$ incubation groups. However, the type 1/type 2 ratio at $30 \mathrm{~min}$ incubation was significantly lower than at 60 or $120 \mathrm{~min}$ of incubation.
Quantitative analysis with a computed analyzer showed a statistically significant difference in reactive products between type 1 and type 2 mitochondria (Table 2). That is, there were many more type 1 mitochondria than type 2 mitochondria in the extraocular muscles.

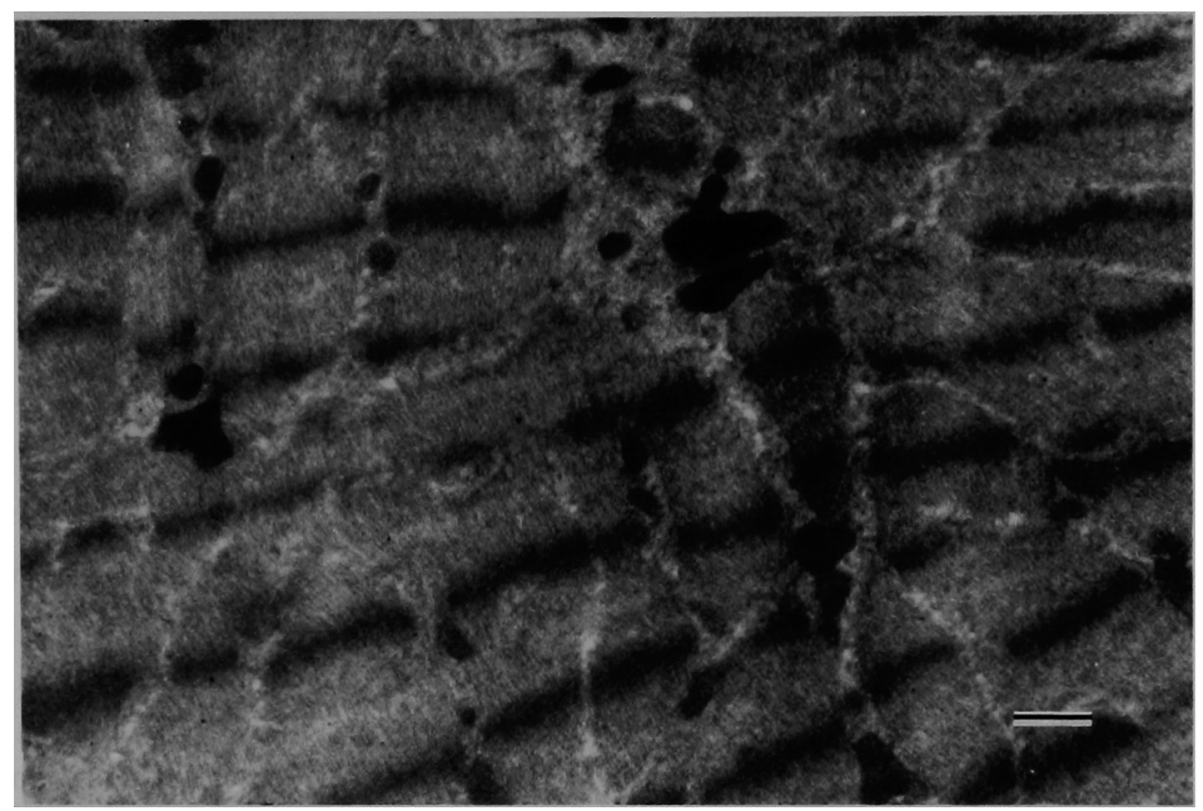

Fig. 2. Electron micrograph of extraocular muscle at $30 \mathrm{~min}$ of incubation. Some mitochondria showed reactive products on the inner membrane, intracristal space and outer compartment. Large number of mitochondria had no reactive products. $\times 8,000$ $\mathrm{Bar}=1 \mu \mathrm{m}$.

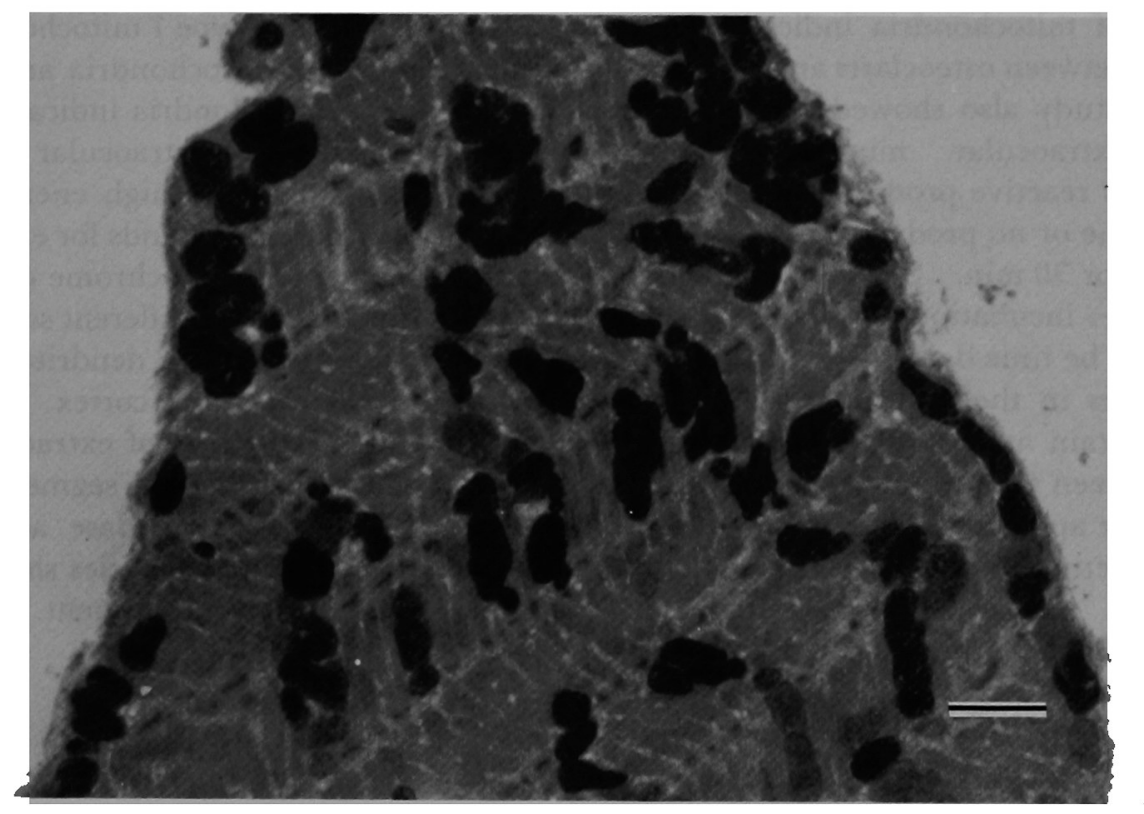

Fig. 3. Electron micrograph of extraocular muscle at $60 \mathrm{~min}$ of incubation. Type 1 mitochondria were increased in number and reactive products filled the intracristal space. $\times 5,000 \quad \mathrm{Bar}=2 \mu \mathrm{m}$. 


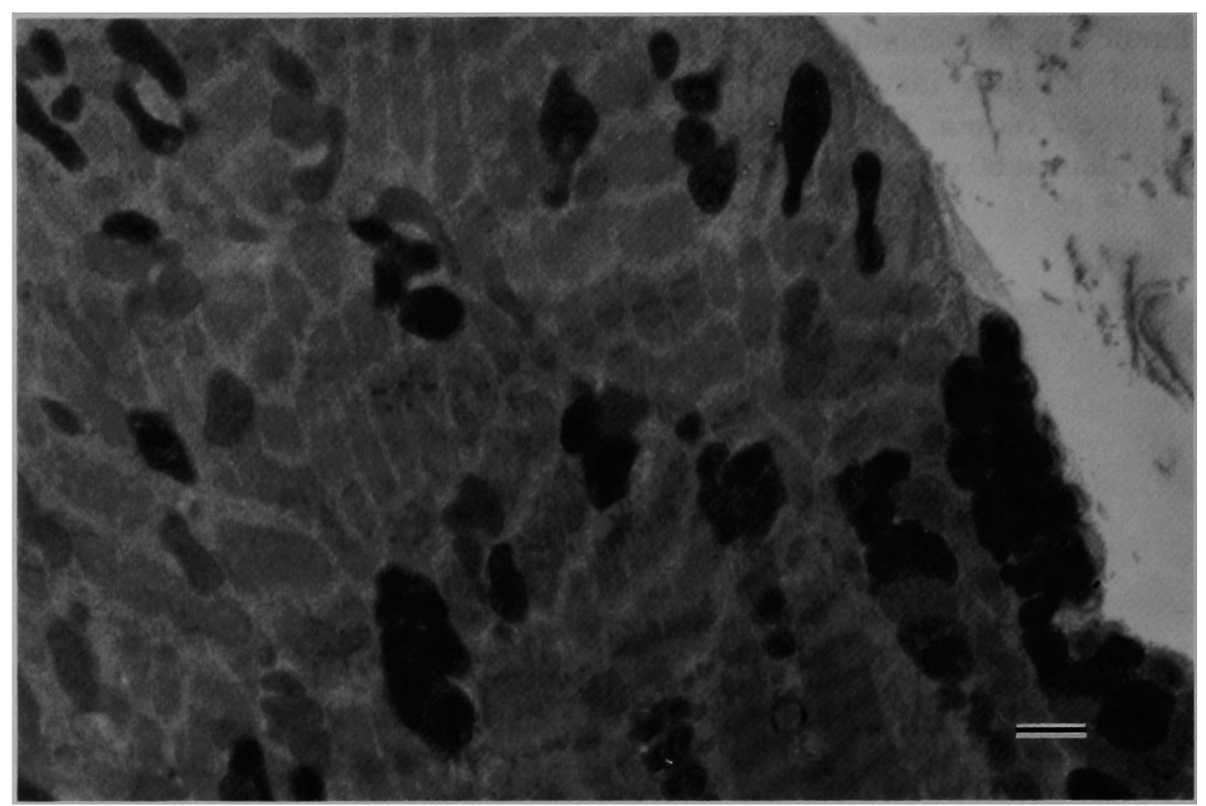

Fig. 4. Electron micrograph of extraocular muscle at $120 \mathrm{~min}$ of incubation. Reactive products in mitochondria were almost same as in those incubated for $60 \mathrm{~min} . \times 6,500 \mathrm{Bar}=2 \mu \mathrm{m}$.

\section{DISCUSSION}

Noda and Kuwahara (4) classified mitochondria into two types and demonstrated that active osteoclasts contain many more type 1 mitochondria, which have reactive products on the inner membrane and in the outer compartment, than preosteoclasts. They concluded that activity of mitochondria indicates functional differentiation between osteoclasts and preosteoclasts. The present study also showed two types of mitochondria in extraocular muscles. Many mitochondria with less reactive products on the inner membrane of the cristae or no products were found in specimens incubated for $30 \mathrm{~min}$. Since these reactive products increased after incubation for 60 or $120 \mathrm{~min}$, the increase seemed to be time dependent. However, there were no changes in the percentage of type 1 mitochondria in a certain area or in the intensity of reactive products between $60 \mathrm{~min}$, and $120 \mathrm{~min}$ of incubation. It therefore appeared that no time-dependent changes of reactive products occurred after $60 \mathrm{~min}$ of incubation and that the two types of mitochondria each had potential cytochrome oxidase activity.

Hirai et al. (1) demonstrated that quantitative analysis with a computed analyzer was useful in the measurement of cytochrome oxidase activity and found different activities of cytochrome oxidase in alveolar type I, II and III cells of the lung.
Extraocular muscles must keep the eyeball constantly static, but they must occasionally move quickly. Thus, slow and quick ocular movements are continuously required of extraocular muscles. In spite of being striated muscle, extraocular muscles are different from general skeletal muscles. In this study, quantitative analysis of muscles incubated for 60 and 120 min showed that type 1 mitochondria had more activity than type 2 mitochondria and that the percentage of type 1 mitochondria indicated the intensity of functional activity in extraocular muscles. Type 1 mitochondria may have high energy and be able to meet the continuous demands for energy and for quick ocular movements. Cytochrome oxidase activity has been reported to vary in different segments of the same dendrite and between the dendrites and soma of the same cell in the cat striate cortex. The present study examined only the caput of extraocular muscles and did not compare different segments of extraocular muscles. Cytochrome oxidase activity in different segments of extraocular muscles should be the subject of another interesting experiment.

\section{REFERENCES}

1. Hirai, K., Ogawa, K., Wang, G. Y. and Ueda, T.: Varied cytochrome oxidase activities of the alveolar type I, type II and type III cells in rat lung: Quantitative 
cytochemistry. J. Electron Microsc. 38; 449-456, 1989.

2. Kageyama, G. and Wong-Rilay, M.: An analysis of cellular localization of cytochrome oxidase in the lateral geniculate nucleus of the adult cat. J. Comp. Neurol. 242; 338-357, 1985.

3. Kageyama, G. and Wong-Rilay, M.: Laminar and cellular localization of the cytochrome oxidase in the cat striate cortex. J. Comp. Neurol. 245; 137-159, 1986.
4. Noda, K. and Kuwahara, Y.: Cytochrome oxidase activity in preosteoclasts induced by experimental tooth movement. J. Electron Microsc. 39; 97-100, 1990.

5. Seligman, A. M., Karnovsky, M. J., Wasserkrung, H. L. and Hanker, J.S.: Nondroplet ultrastructural demonstration of cytochrome oxidase activity with a polymerizing osmiophilic reagent, diaminobenzidine (DAB). J. Cell Biol. 38; 1-14, 1968. 